\title{
On Meager Additive and Null Additive Sets in the Cantor Space $2^{\omega}$ and in $\mathbb{R}$
}

by

\author{
Tomasz WEISS \\ Presented by Czesław RYLL-NARDZEWSKI
}

Summary. Let $T$ be the standard Cantor-Lebesgue function that maps the Cantor space $2^{\omega}$ onto the unit interval $\langle 0,1\rangle$. We prove within ZFC that for every $X \subseteq 2^{\omega}, X$ is meager additive in $2^{\omega}$ iff $T(X)$ is meager additive in $\langle 0,1\rangle$. As a consequence, we deduce that the cartesian product of meager additive sets in $\mathbb{R}$ remains meager additive in $\mathbb{R} \times \mathbb{R}$. In this note, we also study the relationship between null additive sets in $2^{\omega}$ and $\mathbb{R}$.

1. Introduction. Assume that $\left(2^{\omega}, \oplus\right)$ denotes the Cantor space $2^{\omega}$ with modulo 2 coordinatewise addition, and $\mathbb{R}$ is the additive group of real numbers, both with the standard topology and measure. We will say that $X \subseteq 2^{\omega}$ is meager additive (respectively, null additive) iff for every meager (respectively, null) set $A, X \oplus A=\{x \oplus a: x \in X, a \in A\}$ is meager (respectively, null) in $2^{\omega}$. Analogously we define meager additive and null additive sets in $\mathbb{R}$. The following question was asked by T. Bartoszyński (personal communication): Suppose that there exists an uncountable meager (respectively, null) additive set in $\left(2^{\omega}, \oplus\right)$. Is it true that there is an uncountable meager (respectively, null) additive set in $\mathbb{R}$ ? And how about the converse implication?

We give a complete answer (in ZFC) to the category part of this question, and we partially answer the measure version.

To start, let us notice that $2^{\omega}$ can be related to the interval $\langle 0,1\rangle$ through the Cantor-Lebesgue continuous function $T: 2^{\omega} \rightarrow\langle 0,1\rangle$ given by

$$
T(x)=\sum_{i \in \omega} \frac{x(i)}{2^{i+1}} .
$$

2010 Mathematics Subject Classification: 03E05, 03E15, 03E35.

Key words and phrases: meager additive sets, null additive sets, translations in $2^{\omega}$ and in $\mathbb{R}$. 
It is well known that $T$ is category and measure preserving, and oneto-one except on the countable set of sequences that are eventually zero (respectively, one). Thus depending on the context, a subset $X$ of $2^{\omega}$ is often identified with $T(X)=\{T(x): x \in X\}$, and $Y \subseteq\langle 0,1\rangle$ is identified with $T^{-1}(Y)$. Let us also notice that instead of using meager (respectively, null) additive sets in $\mathbb{R}$, we may consider meager (respectively, null) additive sets in $\left(\langle 0,1),+_{1}\right)$, where $+_{1}$ denotes modulo 1 addition. Clearly, the latter sets are meager (respectively, null) additive in $\left(\langle 0,1\rangle,+_{1}\right)$, where $x+{ }_{1} y=x+y$ if $x+y \leq 1$, and $x+{ }_{1} y=x+y-1$ if $x+y>1$. Conversely, if $X$ is a meager (respectively, null) additive set in $\left(\langle 0,1\rangle,+_{1}\right)$, then $X \backslash\{1\}$ is meager (respectively, null) additive in $\left(\langle 0,1),+_{1}\right)$.

We use standard terminology and notation. $\omega^{\omega \uparrow}$ stands for the set of all increasing functions $f: \omega \rightarrow \omega$. For $n \in \omega$ and $f \in \omega^{\omega \uparrow}$,

$$
[f(n), f(n+1))=\{k \in \omega: f(n) \leq k<f(n+1)\},
$$

and if $s \in 2^{[f(n), f(n+1))}$, then

$$
[s]=\left\{x \in 2^{\omega}: x \uparrow[f(n), f(n+1))=s\right\} .
$$

The quantifiers $\exists^{\infty} n, \forall \forall^{\infty} n$ denote "for infinitely many $n$ " and "for all but finitely many $n "$, respectively.

Suppose that $f \in \omega^{\omega \uparrow}$ and $x \in 2^{\omega}$. We define a meager set in $2^{\omega}$ by

$$
B_{f, x}=\left\{y \in 2^{\omega}: \forall^{\infty} n x\lceil[f(n), f(n+1)) \neq y\lceil[f(n), f(n+1))\} .\right.
$$

It is well known (see [BJ, Theorem 2.2.4, p. 10]) that every meager set in $2^{\omega}$ is a subset of a set of the above form, for some $f \in \omega^{\omega \uparrow}$ and $x \in 2^{\omega}$. For $n \in \omega$, we let

$$
B_{[f(n), f(n+1)), x}=\left\{y \in 2^{\omega}: x \uparrow[f(n), f(n+1)) \neq y\lceil[f(n), f(n+1))\},\right.
$$

and we denote by $\mathbb{O}$ (respectively, $\mathbb{1}$ ) the constantly zero (respectively, one) function in $2^{\omega}$. One can easily check that for every $n \in \omega$ and $x \in 2^{\omega}$,

$$
(*) \quad x \oplus B_{[f(n), f(n+1)), \mathbb{O}}=B_{[f(n), f(n+1)), x}=\sum_{i=0}^{f(n+1)-1} \frac{x(i)}{2^{i+1}}+{ }_{1} B_{[f(n), f(n+1)), \mathbb{O}} .
$$

In the identity $(*)$, which plays an important role in the second part of this paper, $+_{1}$ is addition in $\langle 0,1)$, the sets $B_{[f(n), f(n+1)), x}$ and $B_{[f(n), f(n+1)), \mathbb{Q}}$ are identified with their images under $T$, and the sequence of ones $(1,1, \ldots)$ is excluded from the left-hand side of the equation.

\section{Main theorems}

Theorem 1. For every meager additive set $X$ in $\left(2^{\omega}, \oplus\right), T(X)$ is meager additive in $(\langle 0,1\rangle,+1)$. 
Proof. Let $X$ be a meager additive set in $2^{\omega}$. By the Bartoszyński-JudahShelah characterization (see [BJ, Theorem 2.7.17, p. 95]), for every $f \in \omega^{\omega \uparrow}$, there are $g \in \omega^{\omega \uparrow}$ and $y \in 2^{\omega}$ such that

$$
\begin{array}{rl}
\forall x \in X \forall^{\infty} n \exists k g(n) \leq f(k) & <f(k+1)<g(n+1) \text { and } \\
x & x[f(k), f(k+1))=y\lceil[f(k), f(k+1)) .
\end{array}
$$

For $k \in \omega$ and $f \in \omega^{\omega \uparrow}$, let

$$
G_{k}^{f}=\left\{[s]: s \in 2^{[f(k), f(k+1))}\right\} .
$$

Notice that each $[s]$ from $G_{k}^{f}$, treated as a subset of $\langle 0,1\rangle$, is a union of $2^{f(k)}$ intervals of diameter $1 / 2^{f(k+1)}$ each. Define $h_{k}=y\lceil[f(k), f(k+1))$ for $k \in \omega$ and $y \in 2^{\omega}$. Thus, clearly,

$$
\forall f \in \omega^{\omega \uparrow} \exists g \in \omega^{\omega \uparrow} \exists\left\{\left[h_{k}\right]\right\}_{k \in \omega} \forall k \quad\left[h_{k}\right] \in G_{k}^{f},
$$

so that any $x \in X$ belongs to all but finitely many sets of the form

$$
X_{n}^{f}=\bigcup_{k: g(n) \leq f(k)<f(k+1)<g(n+1)}\left[h_{k}\right] .
$$

Claim 2. Let $P$ be a closed nowhere dense subset of $\langle 0,1\rangle$. Given $d>0$ and $k \in \omega$, there are $\varepsilon, \delta>0$ such that for any interval $I$ with $\operatorname{diam}(I) \geq d$, and each set $J$ that consists of $k$ intervals of diameter at most $\varepsilon$, we have

$$
\left(J+{ }_{1} P\right) \cap I^{\prime}=\emptyset,
$$

where $I^{\prime}$ is some interval included in $I$ with $\operatorname{diam}\left(I^{\prime}\right) \geq \delta$.

Proof. It suffices to prove the assertion for a fixed open interval $I$ with $\operatorname{diam}(I)=d>0$, since any interval of diameter no smaller than $d$ can be translated modulo 1 so as to cover $I$. For every $\left\langle x_{0}, \ldots, x_{k-1}\right\rangle \in\langle 0,1\rangle^{k}$, find open intervals $A_{x_{0}}, \ldots, A_{x_{k-1}}$ of equal diameters with middle points $x_{0}, \ldots, x_{k-1}$ such that $\left(A_{x_{0}} \cup \cdots \cup A_{x_{k-1}}\right)+{ }_{1} P$ is disjoint from some open interval $I_{\left\langle x_{0}, \ldots, x_{k-1}\right\rangle} \subseteq I$. Let $\left\{A_{\left\langle x_{0}, \ldots, x_{k-1}\right\rangle}\right\}_{\left\langle x_{0}, \ldots, x_{k-1}\right\rangle \in\langle 0,1\rangle^{k}}$ be the open cover of $\langle 0,1\rangle^{k}$ that consists of the cartesian products of the middle thirds of the sets $A_{x_{0}}, \ldots, A_{x_{k-1}}$. Use compactness of $\langle 0,1\rangle^{k}$ to choose $\varepsilon$ and $\delta$.

Let $P$ be a closed nowhere dense subset of $\langle 0,1\rangle$. We construct $f \in \omega^{\omega \uparrow}$ and a sequence $\left\{d_{n}\right\}_{n \in \omega}$ of positive real numbers iteratively using Claim 2 . Set $d_{0}=1 / 2, f(0)=0$, and assume that $f(n), d_{n}$ have already been constructed. Define $f(n+1)$ so that for any interval $I$ with $\operatorname{diam}(I) \geq d_{n} / 2^{n}$, and any finite set $J$ consisting of at most $2^{f(n)}$ intervals of diameter at most $1 / 2^{f(n+1)}$ each, $J+{ }_{1} P$ is disjoint from a certain interval $I^{\prime} \subseteq I$ with $\operatorname{diam}\left(I^{\prime}\right) \geq d_{n+1}$.

Now let $\left\{I_{n}\right\}_{n \in \omega}$ be a fixed bijective enumeration of all rational open intervals in $\langle 0,1\rangle$, and suppose that $g \in \omega^{\omega \uparrow}$ and $\left\{\left[h_{k}\right]\right\}_{k \in \omega}$ are chosen for $f$ as in the Bartoszyński-Judah-Shelah characterization. Set $\bar{d}_{0}=\operatorname{diam}\left(I_{0}\right)$. 
Since $d_{n} / 2^{n} \rightarrow 0$, we can pick $k_{0}$ so large that $d_{k_{0}} / 2^{k_{0}} \leq \bar{d}_{0}$. Let $n_{0} \geq 0$ be the maximum $n$ satisfying $g(n) \leq f\left(k_{0}\right)$. Using the properties of the function $f$ defined above, we choose a sequence of open intervals $\left\{I_{0}^{k}\right\}_{k: g\left(n_{0}\right) \leq f(k)<f(k+1)<g\left(n_{0}+1\right)}$ so that

$$
\begin{aligned}
& \left(\left[h_{k_{0}}\right]+{ }_{1} P\right) \cap I_{0}^{k_{0}}=\emptyset, \quad I_{0}^{k_{0}} \subseteq I_{0}, \\
& \left(\left[h_{k_{0}+1}\right]+{ }_{1} P\right) \cap I_{0}^{k_{0}+1}=\emptyset, \quad I_{0}^{k_{0}+1} \subseteq I_{0}^{k_{0}}, \quad \text { etc. }
\end{aligned}
$$

We let $I_{0}^{\prime}=\bigcap_{j: f\left(k_{0}+j+1\right)<g\left(n_{0}+1\right)} I_{0}^{k_{0}+j}$. Then

$$
\left(\left(\bigcup_{k: g\left(n_{0}\right) \leq f(k)<f(k+1)<g\left(n_{0}+1\right)}\left[h_{k}\right]\right)+{ }_{1} P\right) \cap I_{0}^{\prime}=\emptyset .
$$

Now set $\bar{d}_{1}=\operatorname{diam}\left(I_{1}\right)$. As before, we choose $k_{1}$ with $d_{k_{1}} / 2^{k_{1}} \leq \bar{d}_{1}$, and we pick $n_{1}$ satisfying $g\left(n_{1}\right)>g\left(n_{0}+1\right)$, and a sequence of open intervals $\left\{I_{1}^{k}\right\}_{k: g\left(n_{1}\right) \leq f(k)<f(k+1)<g\left(n_{1}+1\right)}$. Let $I_{1}^{\prime}=\bigcap_{j: f\left(k_{1}+j+1\right)<g\left(n_{1}+1\right)} I_{1}^{k_{1}+j}$. Then

$$
\left(\left(\bigcup_{k: g\left(n_{1}\right) \leq f(k)<f(k+1)<g\left(n_{1}+1\right)}\left[h_{k}\right]\right)+{ }_{1} P\right) \cap I_{1}^{\prime}=\emptyset .
$$

We follow this scenario for $I_{2}, I_{3}, \ldots$ etc.

Finally, we conclude that

$$
\bigcap_{j \in \omega}\left(\bigcup_{k: g\left(n_{j}\right) \leq f(k)<f(k+1)<g\left(n_{j}+1\right)}\left[h_{k}\right]\right)+{ }_{1} P
$$

is meager. Thus $\bigcap_{n \geq 0} X_{n}^{f}+{ }_{1} P$ is meager. In a similar way we show that $\bigcap_{n \geq m} X_{n}^{f}+{ }_{1} P$ is meager for all $m \geq 1$. This finishes the proof of Theorem 1 .

Corollary 3. Suppose that $X, Y$ are meager additive sets in $2^{\omega}$. Then their cartesian product is meager additive in $\left(\langle 0,1\rangle,+_{1}\right) \times\left(\langle 0,1\rangle,+_{1}\right)$.

Proof. First notice that one can apply the same argument as in the proof of Theorem 1 to show that $X \times\{0\}$ and $\{0\} \times Y$ are meager additive in the product $\left(\langle 0,1\rangle,+_{1}\right) \times\left(\langle 0,1\rangle,+_{1}\right)$ with modulo 1 coordinatewise addition (denoted by + ). Then use the fact that for any meager set $A$ in the product, $X \times Y+A=X \times\{0\}+(\{0\} \times Y+A)$ is meager.

TheOREM 4. Let $X$ be meager additive in $\left(\langle 0,1),+_{1}\right)$. Then there exists $t \in\langle 0,1)$ such that $T^{-1}\left(X+{ }_{1} t\right)$ is meager additive in $\left(2^{\omega}, \oplus\right)$.

Proof. Our goal is to show that for any meager additive set $X$ in $\left(\langle 0,1),+_{1}\right)$, there exists $t \in\langle 0,1)$ such that for every meager set $A$ in $2^{\omega}$, one can find a meager set $B$ in $\langle 0,1)$ satisfying

$$
T\left(T^{-1}\left(X+{ }_{1} t\right) \oplus A\right) \subseteq\left(X+{ }_{1} t\right)+{ }_{1} B .
$$


Claim 5. Let $F$ be an $F_{\sigma}$ meager subset of $\langle 0,1)$. Then there is $t \in\langle 0,1)$ such that $\left(F+{ }_{1} t\right) \cap Q=\emptyset$, where $Q$ is the set of all rational numbers in $\langle 0,1)$.

Proof. Let $t$ be such that $-{ }_{1} t \notin F+{ }_{1} Q$.

Claim 6. Suppose that $X$ is a meager additive subset of $\langle 0,1)$. Then there is a $\sigma$-compact set $F$ disjoint from $Q$ such that $X+{ }_{1} t \subseteq F$ for some $t \in\langle 0,1)$.

Proof. Since $X$ is meager, there exists a meager $F_{\sigma}$ set $\widetilde{F}$ such that $X \subseteq \widetilde{F}$. Apply Claim 5 to find $t$ with $\left(\widetilde{F}+{ }_{1} t\right) \cap Q=\emptyset$, and then put $F=\widetilde{F}+{ }_{1} t$. Notice that we can assume that $F$ is a $\sigma$-compact subset of $\langle 0,1)$.

Claim 7. Suppose that $F$ is a $\sigma$-compact subset of $\langle 0,1)$ disjoint from $Q$, and $h \in \omega^{\omega \uparrow}$. Then there is an increasing sequence $\left\{n_{k}\right\}_{k \in \omega}$ of natural numbers such that

$$
F \subseteq\left\{x \in 2^{\omega}: \forall^{\infty} k x \uparrow\left[h\left(n_{k}\right), h\left(n_{k+1}\right)\right) \neq \mathbb{1}\right\} .
$$

Proof. We define a continuous function $g: F \rightarrow \omega^{\omega}$ as follows. For $x \in F$, we let $g(x)(0)=h(0), g(x)(n)=\min \{m: m>\max \{h(n), n\}, m=h(k)$ for some $k \in \omega$, and $x \uparrow[g(x)(n-1), g(x)(m)) \neq \mathbb{1}\}$. As $g$ is continuous, its range is a $\sigma$-compact subset of $\omega^{\omega}$, thus there exists $G \in \omega^{\omega \uparrow}$, with range $(G) \subset$ range $(h)$, such that for every $x \in F$,

$$
\exists n_{0} \forall k \geq n_{0} g(x)(k) \leq G(k) .
$$

For $k \in \omega$, let $G^{k}$ be the $k$-fold iteration of $G$. We define $n_{k}$ to be the $n$ such that $h(n)=G^{2 k}(0)$, for $k \in \omega$.

Claim 8. Assume that $k, n \in \omega, n \geq 2$ and $f \in \omega^{\omega \uparrow}$. If $X \subseteq 2^{\omega}$ and $x \uparrow[f(k+1), f(k+n)) \neq \mathbb{1}$ for every $x \in X$, then

$$
X \oplus B_{[f(k), f(k+1)), \mathbb{Q}} \subseteq X+{ }_{1} B_{[f(k), f(k+n)), \mathbb{O}} .
$$

Proof. Fix $s \in 2^{f(k)}$ and identify $B_{[f(k), f(k+1)), \mathbb{Q}}$ and $B_{[f(k), f(k+n)) \text {, } \mathbb{O} \text { with }}$ their images under $T$. Then the distance between the left endpoint of the interval $\left\{y \in 2^{\omega}: y\left\lceil f(k)=s, y \in B_{[f(k), f(k+n)), \mathbb{O}\}}\right.\right.$ and the left endpoint of the interval $\left\{y \in 2^{\omega}: y\left\lceil f(k)=s, y \in B_{[f(k), f(k+1)), \mathbb{O}}\right\}\right.$ is equal to $1 / 2^{f(k+1)}$ $1 / 2^{f(k+n)}$. Since $x\lceil[f(k+1), f(k+n)) \neq \mathbb{1}$, we have

$$
\sum_{i=f(k+1)}^{\infty} \frac{x(i)}{2^{i+1}} \leq \frac{1}{2^{f(k+1)}}-\frac{1}{2^{f(k+n)}} .
$$

Thus $B_{[f(k), f(k+1)), \mathbb{Q}}$ remains included in the modulo $1($ in $\langle 0,1))$ translation 
of $B_{[f(k), f(k+n)), \mathbb{O}}$ by $\sum_{i=f(k+1)}^{\infty} x(i) / 2^{i+1}$, that is,

$$
B_{[f(k), f(k+1)), \mathbb{O}} \subseteq \sum_{i=f(k+1)}^{\infty} \frac{x(i)}{2^{i+1}}+{ }_{1} B_{[f(k), f(k+n)), \mathbb{O}} .
$$

By the identity $(*)$ from Section 1 , we obtain

$$
\begin{aligned}
B_{[f(k), f(k+1)), x} & =\sum_{i=0}^{f(k+1)-1} \frac{x(i)}{2^{i+1}}+{ }_{1} B_{[f(k), f(k+1)), \mathbb{O}} \\
& \subseteq \sum_{i=0}^{f(k+1)-1} \frac{x(i)}{2^{i+1}}+{ }_{1} \sum_{i=f(k+1)}^{\infty} \frac{x(i)}{2^{i+1}}+{ }_{1} B_{[f(k), f(k+n)), \mathbb{O}} \\
& =x+{ }_{1} B_{[f(k), f(k+n)), \mathbb{O}} \cdot
\end{aligned}
$$

Now assume that $X$ is meager additive in $\left(\langle 0,1),+_{1}\right)$, and let $t$ be such that $\left(X+{ }_{1} t\right) \cap Q=\emptyset$. Set $X_{m}=\left\{x \in 2^{\omega}: x \in X+_{1} t\right.$ and $\forall k \geq m$ $x\left\lceil\left[h\left(n_{k}\right), h\left(n_{k+1}\right)\right) \neq \mathbb{1}\right\}$ for $m \in \omega$, where $\left\{n_{k}\right\}_{k \in \omega}$ is chosen for a given $h \in \omega^{\omega \uparrow}$ as in Claim 7. Obviously, $X+{ }_{1} t=\bigcup_{m \in \omega} X_{m}$. Let $m_{0} \in \omega$ be fixed. Suppose that $x \in X_{m_{0}}$. Then for every $m \geq m_{0}$,

$$
\begin{aligned}
x \oplus \bigcap_{n \geq m} B_{[h(n), h(n+1)), \mathbb{O}} & =\bigcap_{\substack{n \geq m\\
}}\left(x \oplus B_{[h(n), h(n+1)), \mathbb{O}}\right) \\
& \subseteq \bigcap_{\substack{k \geq m \\
k \text { even }}}\left(x \oplus B_{\left[h\left(n_{k}\right), h\left(n_{k+1}\right)\right), \mathbb{O}}\right) .
\end{aligned}
$$

By Claim 8, for every $n_{k} \geq m$,

$$
x \oplus B_{\left[h\left(n_{k}\right), h\left(n_{k+1}\right)\right), \mathbb{O}}=B_{\left[h\left(n_{k}\right), h\left(n_{k+1}\right)\right), x} \subseteq x+{ }_{1} B_{\left[h\left(n_{k}\right), h\left(n_{k+2}\right)\right), \mathbb{O}} .
$$

It follows that

$$
\begin{aligned}
x \oplus \bigcap_{n \geq m} B_{[h(n), h(n+1)), \mathbb{O}} & \subseteq \bigcap_{\substack{k \geq m \\
k \text { even }}}\left(x+{ }_{1} B_{\left[h\left(n_{k}\right), h\left(n_{k+2}\right)\right), \mathbb{O}}\right) \\
& =x+1 \bigcap_{\substack{k \geq m \\
k \text { even }}} B_{\left[h\left(n_{k}\right), h\left(n_{k+2}\right)\right), \mathbb{O}} .
\end{aligned}
$$

Consequently,

$$
X_{m_{0}} \oplus \bigcap_{n \geq m} B_{[h(n), h(n+1)), \mathbb{O}} \subseteq X_{m_{0}}+1 \bigcap_{\substack{k \geq m \\ k \text { even }}} B_{\left[h\left(n_{k}\right), h\left(n_{k+2}\right)\right), \mathbb{O}} .
$$

As $\bigcap_{k \geq m, k \text { even }} B_{\left[h\left(n_{k}\right), h\left(n_{k+2}\right)\right), \mathbb{O}}$ is closed nowhere dense, the right hand side above remains meager. Hence the image of $X_{m_{0}} \oplus \bigcap_{n \geq m} B_{[h(n), h(n+1)), \mathbb{O}}$ under $T$ is meager in $\langle 0,1)$. Thus $X_{m_{0}} \oplus \bigcap_{n \geq m} B_{[h(n), h(n+1)), \mathbb{O}}$ is meager in $\left(2^{\omega}, \oplus\right)$. 
Consider now any set of the form $B_{h, z}$, where $h \in \omega^{\omega \uparrow}$ and $z \in 2^{\omega}$. Then

$$
X_{m_{0}} \oplus \bigcap_{n \geq m} B_{[h(n), h(n+1)), z}=X_{m_{0}} \oplus \bigcap_{n \geq m} B_{[h(n), h(n+1)), \mathbb{}} \oplus z .
$$

This proves that $T^{-1}\left(X+{ }_{1} t\right)$ is meager additive in $2^{\omega}$.

Corollary 9. Suppose that there is a meager additive set in $\left(\langle 0,1\rangle,+_{1}\right)$ of cardinality $\kappa$, where $\aleph_{0}<\kappa \leq \mathfrak{c}$. Then there exists a meager additive set in $\left(2^{\omega}, \oplus\right)$ of cardinality $\kappa$.

Proof. Apply Theorem 4.

From Theorem 4 the following stronger fact follows immediately.

Theorem 10. For every meager additive set $X$ in $\left(\langle 0,1),+{ }_{1}\right), T^{-1}(X)$ is meager additive in $\left(2^{\omega}, \oplus\right)$.

Proof. Let $X$ be a meager additive set in $\left(\langle 0,1),+_{1}\right)$. Then, by Theorem $4, T^{-1}\left(X+{ }_{1} t\right)$ is meager additive in $2^{\omega}$ for some $t \in\langle 0,1)$.

Let $f \in \omega^{\omega \uparrow}$. As in the first part of this paper, let $g \in \omega^{\omega \uparrow}$ and $\left\{\left[h_{k}\right]\right\}_{k \in \omega}$, with each $h_{k} \in G_{k}^{f}$, be such that any $x$ from $X+{ }_{1} t$ belongs to almost every set of the form

$$
\bigcup_{k: g(n) \leq f(k)<f(k+1)<g(n+1)}\left[h_{k}\right] .
$$

Then there are $\left\{\left[h_{k}^{\prime}\right]\right\}_{k \in \omega}$ and $\left\{\left[h_{k}^{\prime \prime}\right]\right\}_{k \in \omega}$, with $h_{k}^{\prime}, h_{k}^{\prime \prime} \in G_{k}^{f}$ for $k \in \omega$, such that any $x \in X$ belongs to almost every set of the form

$$
\bigcup_{k: g(n) \leq f(k)<f(k+1)<g(n+1)}\left[h_{k}^{\prime}\right] \cup\left[h_{k}^{\prime \prime}\right] .
$$

Thus, by applying Claim 2 for subsets of $2^{\omega}$, we can proceed as in the proof of Theorem 1 to show that $T^{-1}(X)$ is meager additive in $2^{\omega}$.

Remark 11. Notice that by Corollary 3 and Theorem 10, the cartesian product of meager additive sets in $\left(\langle 0,1\rangle,+_{1}\right)$ is meager additive in $\left(\langle 0,1\rangle,+_{1}\right) \times\left(\langle 0,1\rangle,+_{1}\right)$. This can be easily extended to products of meager additive subsets of $\mathbb{R}$ (see Problem 2.4 and Remark 2.5 in [TW]).

Unfortunately, we do not know if one can establish a result analogous to Theorem 4 for null additive sets in $\left(\langle 0,1),+_{1}\right)$. So the following crucial question remains open.

QUESTION 12. Suppose that there is an uncountable null additive set in $\left(\langle 0,1),+_{1}\right)$. Does this imply that its "reasonable" transformation is null additive in $2^{\omega}$ ?

Nevertheless, the following theorem holds. 
Theorem 13. Suppose that $X$ is null additive in $\left(2^{\omega}, \oplus\right)$. Then it is null additive in $(\langle 0,1\rangle,+1)$.

Proof. By Shelah's characterization (see [BJ, Theorem 2.7.18(3), p. 95]), for every $f \in \omega^{\omega \uparrow}$, there is a sequence $\left\{I_{n}\right\}_{n \in \omega}$, with each $I_{n} \subseteq 2^{[f(n), f(n+1))}$ and $\left|I_{n}\right| \leq n$, such that

$$
\forall x \in X \forall^{\infty} n x \uparrow[f(n), f(n+1)) \in I_{n} .
$$

Let $H$ be a null set in $\langle 0,1\rangle$. Then, by Bartoszyński's theorem, $H$, treated as a subset of $2^{\omega}$, is contained in a union of two small sets (see [BJ, Theorem 2.5.7, p. 63]). Recall that $A \subseteq 2^{\omega}$ is small if there are $f \in \omega^{\omega \uparrow}$ and a sequence $\left\{J_{n}\right\}_{n \in \omega}$, with $J_{n} \subseteq 2^{[f(n), f(n+1))}$, such that

$$
\begin{gathered}
A \subseteq\left\{x \in 2^{\omega}: \exists^{\infty} n x \uparrow[f(n), f(n+1)) \in J_{n}\right\}, \\
\sum_{n \in \omega} \frac{\left|J_{n}\right|}{2^{f(n+1)-f(n)}}<\infty .
\end{gathered}
$$

Thus we may assume for the purpose of this proof that there is $f \in \omega^{\omega \uparrow}$ such that

$$
H=\left\{\sum_{i \in \omega} \frac{x(i)}{2^{i+1}}: x \in 2^{\omega} \text { and } \exists^{\infty} n x\left\lceil[f(n), f(n+1)) \in J_{n}\right\},\right.
$$

where each $J_{n} \subseteq 2^{[f(n), f(n+1))}$ and

$$
\forall n \in \omega \frac{\left|J_{n}\right|}{2^{f(n+1)-f(n)}} \leq \frac{1}{2^{n}}
$$

By Shelah's characterization, $X$, as a subset of $\langle 0,1\rangle$, satisfies

$$
X \subseteq \bigcup_{k \in \omega} \bigcap_{n \geq k} X_{n}
$$

where for $n \in \omega$,

$$
X_{n}=\left\{\sum_{i \in \omega} \frac{x(i)}{2^{i+1}}: x \in 2^{\omega} \text { and } x\left\lceil[f(n), f(n+1)) \in\left\{I_{1}^{n}, \ldots, I_{n}^{n}\right\}=I_{n}\right\} .\right.
$$

Also,

$$
H \subseteq \bigcap_{k \in \omega} \bigcup_{n \geq k} H_{n}
$$

where for $n \in \omega$,

$$
H_{n}=\left\{\sum_{i \in \omega} \frac{y(i)}{2^{i+1}}: y \in 2^{\omega} \text { and } y\left\lceil[f(n), f(n+1)) \in\left\{J_{1}^{n}, \ldots, J_{r(n)}^{n}\right\}=J_{n}\right\},\right.
$$

with $r(n)=\left|J_{n}\right|$. Clearly,

$$
X+{ }_{1} H \subseteq \bigcap_{k \in \omega} \bigcup_{n \geq k}\left(X_{n}+{ }_{1} H_{n}\right) .
$$


It is easy to see that each $X_{n}+{ }_{1} H_{n}$ is contained in a union of $n \cdot 2^{f(n)} \cdot\left|J_{n}\right|$ intervals of diameter $2 / 2^{f(n+1)}$ each. Thus there is a sequence $\left\{Y_{n}\right\}_{n \in \omega}$ of subsets of $\langle 0,1\rangle$ satisfying

$$
X+{ }_{1} H \subseteq \bigcap_{k \in \omega} \bigcup_{n \geq k} Y_{n}, \quad \mu\left(Y_{n}\right) \leq \frac{2 n \cdot\left|J_{n}\right|}{2^{f(n+1)-f(n)}} \leq \frac{2 n}{2^{n}} .
$$

Since $\sum_{n \in \omega} \frac{2 n}{2^{n}}$ is convergent, $X+{ }_{1} H$ is null.

Added in proof. O. Zindulka has kindly informed us that he knows how to prove Theorems 1 and 13 by substantially different methods. His results will be published elsewhere.

\section{References}

[BJ] T. Bartoszyński and H. Judah, Set Theory, A K Peters, Wellesley, MA, 1995.

[TW] B. Tsaban and T. Weiss, Products of special sets of real numbers, Real Anal. Exchange $30(2004 / 2005), 1-17$.

Tomasz Weiss

Instytut Matematyki

Akademia Podlaska

08-110 Siedlce, Poland

E-mail: tomaszweiss@go2.pl

Received April 9, 2009;

received in final form July 9, 2009 